\title{
Introduction to Mammalian Genome special issue: inflammation and immunity in cancer
}

\author{
Andreas Behren $^{1,2} \cdot$ Daniel Speidel $^{3} \cdot$ George Kollias $^{4,5} \cdot$ Viive M. Howell $^{6,7}$
}

Published online: 2 November 2018

(c) Springer Science+Business Media, LLC, part of Springer Nature 2018

The Hallmarks of Cancer, devised by Hanahan and Weinberg (2000) provided a framework to rationalize the complexity of cancer. This framework has stood the test of time, although was expanded in 2011 from six to ten hallmarks that included the addition of "Tumor-promoting inflammation" and "Avoiding immune destruction" (Hanahan and Weinberg 2011). The hallmarks guide in understanding of the processes and capabilities that enable cancer to develop and progress, and also direct attention to potential targets for the design of treatments.

These hallmarks also refocused attention on the entire tumor rather than just on the cancer cells. Indeed, the tumor microenvironment, and in particular, inflammatory and immune cells, are now areas of intense focus as researchers strive to better understand the factors modulating cancer initiation, progression, metastasis, and response to treatment. However, our understanding is confounded by the complexity and often contradictory functions of these cells. What has become clear is that a highly orchestrated interplay between the different cell types exists and will finally

Viive M. Howell

viive.howell@sydney.edu.au

1 School of Cancer Medicine, La Trobe University, Heidelberg, VIC, Australia

2 Olivia Newton-John Cancer Research Institute, Heidelberg, VIC, Australia

3 Evotec, Hamburg, Germany

4 Division of Immunology, Biomedical Sciences Research Center "Alexander Fleming", Vari, Greece

5 Department of Experimental Physiology, Medical School, National and Kapodistrian University of Athens, Athens, Greece

6 Northern Clinical School, Faculty of Medicine and Health, The University of Sydney, Sydney, NSW, Australia

7 Bill Walsh Translational Cancer Research Laboratory, Kolling Institute, Royal North Shore Hospital, Level 8 Kolling Building, St Leonards, NSW 2065, Australia determine the course of the disease. This special issue grew from the need to give clarity to this complexity, untangle the contradictions, and highlight the pivotal role of these cells and processes in cancer. Further, this issue reviews how this knowledge is being harnessed to design novel treatments for sustained control of particular cancers.

Immunotherapies are now conservatively estimated to be included in at least half of all clinical trials for cancer. While novel approaches are continually being considered, current prominent therapeutic approaches include immune checkpoint inhibitors, therapeutic vaccines, and adoptive cell therapies. Immune checkpoints are key regulators of immune activation that prevent auto-immunity and tissue damage, while at the same time allowing appropriate immune reactions against foreign agents. Inhibition of these checkpoints is a rapidly expanding immunotherapeutic approach to cancer treatment. The success of inhibitors of immune checkpoint molecules CTLA4 and PD-1/PD-L1 has encouraged the investigation of other immune checkpoint molecules. Stamm et al. (2018) review the studies assessing the actions and anti-cancer efficacy of the novel immune checkpoint axis, co-inhibitory receptor T cell immunoreceptor with Ig and ITIM domains (TIGIT), and ligands poliovirus receptor (PVR) and poliovirus receptor-related 2 (PVRL2).

While targeting immune checkpoint molecules on T-cells is a major area of current research, other therapies are also being (re)-evaluated and improved. Therapeutic vaccines have been extensively investigated for the treatment of cancer for several decades, but they have not demonstrated the same successes as immune checkpoint inhibitors. Cebon (2018) reviews the history of therapeutic cancer vaccines, assesses possible reasons for their limited success, and considers the challenges and requirements for designing a vaccine. These vaccines are often based on cancer-testis antigens due to their tissue-restricted expression and Hutchison and Pritchard (2018) review these and other cancer antigens and the recent advances in predicting their activity for improved personalized immunotherapies. $\mathrm{T}$ cell destruction 
of cancer cells in this setting relies mainly on their recognition of specific antigens presented in the context of major histocompatibility complex (MHC), and the relevance of this interaction has been extended beyond human disease. The study of Tasmanian Devil Facial Tumor Disease, a naturally occurring transmissible cancer that almost annihilated the world's Tasmanian Devil population, provides unique and powerful insights into the role of immunity in cancer development. Peel and Belov (2018) describe the studies that led to the discovery that low genetic diversity of the MHC in combination with other immune factors enabled the disease to evade immune recognition and be transmitted, essentially as an allograft with complete penetrance. They go on to review immunization and immunotherapy as avenues of promise for the treatment for this immune-related malignancy.

Another T cell-based strategy to target cancer is the use of adoptive cell transfer (ACT) of T-cells genetically modified to express synthetic chimeric antigen receptors (CAR T-cells). This approach has revolutionized the treatment of some hematological malignancies with two agents now approved in USA and many in clinical trials. However, the same dramatic and long-term responses have not been seen in solid tumors to date. Gowrishankar et al. (2018) review current and next-generation adoptive $\mathrm{T}$ cell therapies and their production. They also consider the barriers inherent to the tumor microenvironment that limit the success of these therapies in solid tumors.

Clearly, for vaccines and ACT strategies, identifying the right target is a critical step. Hayes et al. (2018) review classical, serological, and mass spectrometric methods for proteomic analysis and discuss how the emerging area of immunoproteomics is informing immunotherapy design and monitoring. While T-cells have held (and still hold) the limelight in immuno-oncology and as therapeutic targets, dysregulation in their differentiation and development can also be linked to increased cancer risk. Ginn et al. (2018) review the controversial area of thymocyte renewal and oncogenic risk and provide their perspective on whether these studies have relevance to the treatment of immunodeficiency disorders in humans. Obviously, T-cells are not acting in isolation, and other immune cells have key roles in immune regulation, balance, and disease as well. Innate immune cells are the so-called 'first responders' in infectious disease, but their role in cancers remains under researched. Here, Guillerey (2018) unravels the complexity of innate lymphoid cells in cancer and reviews the duality of these cells, comparing the pro- and anti-tumor functions that have been reported and their potential as targets for cancer immunotherapeutics.

Da Gama Duarte et al. (2018) draw attention to B cells and their cellular and humoral roles in melanoma. They review the possible mechanisms of activation and roles of $\mathrm{B}$ cells and the antibodies they produce in early anti-tumor immune surveillance. Given the prognostic significance of immune cells within the tumor microenvironment, they consider the potential utility of B cells and their antibodies as diagnostic, prognostic, and predictive biomarkers for melanoma.

Given those data, activation of the immune system in response to cancer or foreign stimuli seems to be the adequate response of the body. But, as with all biological systems, a very fine line exists between appropriate stimulation and dysbalance. Cho et al. (2018) provide an overview of the inflammation pathway and how it joins forces with the coagulation pathway to protect the body against foreign assault. They describe how, when inflammation is dysregulated, an imbalance in coagulation favors a pro-coagulant state. They detail these changes in high-grade glioma, review how these impact tumor behavior, and speculate on potential therapies targeting these pathways.

Perera at al. (2018) focus on chronic inflammation in the gastrointestinal tract (colitis) and how this may lead to colorectal cancer. They highlight diet and the microbiome as key environmental triggers of intestinal inflammation and review the role of the NLRP3 inflammasome in modulating these triggers in colitis. Kazantseva et al. (2018) link inflammation in cancer with p53 isoforms. While p53 is best known for its central role in the arrest of cell division when DNA damage is detected, the discovery of naturally occurring isoforms expanded its mechanistic repertoire. Kazantseva et al. (2018) review the pathologies of the $\Delta 122 \mathrm{p} 53$ mouse (a model of the human $\Delta 133$ p53 isoform) detailing the proinflammatory and autoimmune findings in addition to the established tumor-promoting properties. They compare these with findings from human studies and propose that the isoforms evolved as pro-inflammatory molecules to counter the damage to tissue following p53 activation.

As our understanding of the roles of immune response and inflammation in the etiology of cancer increases, so too increases our understanding of the roles these processes may play in treating cancer. While radiation has been used as a treatment for cancer since 1900, its roles in eliciting inflammatory and immune responses and enhancing the effects of immunotherapies are only now starting to be considered and exploited. McKelvey et al. (2018) discuss how ionizing radiation initiates and modulates inflammatory and immune responses and how these may be harnessed for optimal treatment.

The question that emerges is how can we as researchers and clinicians integrate this expanding knowledge and optimally utilize it to monitor and visualize at least parts of the tumor immune microenvironment to understand the interactions that determine therapy responses? Lim and Rizos (2018) review the technologies that researchers now have at their disposal, including flow cytometry, immunohistochemistry, both single color and multispectral, mass 
cytometry for single-cell proteomics and transcriptomics, in addition to proteomics. They discuss these as aids to understand response to immunotherapy as well as the mechanisms of resistance, a rapidly expanding area of intense research focus in immuno-oncology.

We would like to thank all of the authors for their contributions to this special issue and Managing Editor Louise Tinsley for her patient but efficient managing of all the authors and editors. We hope you enjoy reading this special issue.

\section{References}

Cebon J (2018) Perspective: cancer vaccines in the era of immune checkpoint blockade. Mamm Genome 29. https://doi.org/10.1007/ s00335-018-9786-z

Cho A, McKelvey KJ, Lee A, Hudson AL (2018) The intertwined fates of inflammation and coagulation in glioma. Mamm Genome 29. https://doi.org/10.1007/s00335-018-9761-8

Da Gama Duarte J, Peyper JM, Blackburn JM (2018) B cells and antibody production in melanoma. Mamm Genome 29. https://doi. org/10.1007/s00335-018-9778-z

Ginn SL, McCormack MP, Alexander IE (2018) Thymocyte selfrenewal and oncogenic risk in immunodeficient mouse models: relevance for human gene therapy clinical trials targeting haematopoietic stem cell populations? Mamm Genome 29. https://doi. org/10.1007/s00335-018-9780-5

Gowrishankar K, Birtwistle L, Micklethwaite K (2018) Manipulating the tumor microenvironment by adoptive cell transfer of CAR T-cells. Mamm Genome 29. https://doi.org/10.1007/s0033 5-018-9756-5
Guillerey C (2018) Roles of cytotoxic and helper innate lymphoid cells in cancer. Mamm Genome 29. https://doi.org/10.1007/s0033 5-018-9781-4

Hanahan D, Weinberg RA (2000) The hallmarks of cancer. Cell 100:57-70

Hanahan D, Weinberg RA (2011) Hallmarks of cancer: the next generation. Cell 144:646-674. https://doi.org/10.1016/j.cell.2011.02.013

Hayes SA, Clarke S, Pavlakis N, Howell VM (2018) The role of proteomics in the age of immunotherapies. Mamm Genome 29. https ://doi.org/10.1007/s00335-018-9763-6

Hutchison S, Pritchard AL (2018) Identifying neoantigens for use in immunotherapy. Mamm Genome 29. https://doi.org/10.1007/ s00335-018-9771-6

Kazantseva M, Mehta S, Eiholzer RA, Hung N, Wiles A, Slatter TL, Braithwaite AW (2018) A mouse model of the Delta133p53 isoform: roles in cancer progression and inflammation. Mamm Genome 29. https://doi.org/10.1007/s00335-018-9758-3

Lim SY, Rizos H (2018) Immune cell profiling in the age of immune checkpoint inhibitors: implications for biomarker discovery and understanding of resistance mechanisms. Mamm Genome 29. https://doi.org/10.1007/s00335-018-9757-4

McKelvey KJ, Hudson AL, Back M, Eade T, Diakos CI (2018) Radiation, inflammation and the immune response in cancer. Mamm Genome 29. https://doi.org/10.1007/s00335-018-9777-0

Peel E, Belov K (2018) Lessons learnt from the Tasmanian devil facial tumour regarding immune function in cancer. Mamm Genome 29. https://doi.org/10.1007/s00335-018-9782-3

Perera AP, Sajnani K, Dickinson J, Eri R, Korner H (2018) NLRP3 inflammasome in colitis and colitis-associated colorectal cancer. Mamm Genome 29. https://doi.org/10.1007/s00335-018-9783-2

Stamm H, Wellbrock J, Fiedler W (2018) Interaction of PVR/PVRL2 with TIGIT/DNAM-1 as a novel immune checkpoint axis and therapeutic target in cancer. Mamm Genome 29. https://doi. org/10.1007/s00335-018-9770-7 